University of New Mexico

UNM Digital Repository

Mathematics and Statistics Faculty and Staff

Publications

Academic Department Resources

2020

Interval Valued Neutrosophic Shortest Path Problem by A* Algorithm

Florentin Smarandache

S. Khrisna Prabha

Said Broumi

Follow this and additional works at: https://digitalrepository.unm.edu/math_fsp

Part of the Discrete Mathematics and Combinatorics Commons, Logic and Foundations Commons, Other Mathematics Commons, and the Set Theory Commons 


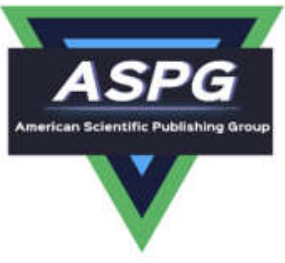

\title{
Interval Valued Neutrosophic Shortest Path Problem by
}

\section{A* Algorithm}

\author{
${ }^{1}$ S.Krishna Prabha, ${ }^{2}$ Said Broumi, ${ }^{3}$ Florentin Smarandache \\ 1 Assistant Professor, Department of Mathematics, \\ PSNA College of Engineering and Technology, Dindigul-624622, Tamilnadu, India;,jvprbh1@gmail.com \\ ${ }^{2}$ Laboratory of Information Processing, Faculty of Science Ben M'Sik, \\ University Hassan II, Casablanca, Morocco ;broumisaid78@gmail.com \\ ${ }^{3}$ Dept. Math and Sciences, University of New Mexico, Gallup, NM, USA; smarand@unm.edu \\ * Correspondence: jvprbh1@gmail.com
}

\begin{abstract}
Many researchers have been proposing various algorithms to unravel different types of fuzzy shortest path problems. There are many algorithms like Dijkstra's, Bellman-Ford,Floyd-Warshall and kruskal's etc are existing for solving the shortest path problems. In this work a shortest path problem with interval valued neutrosophic numbers is investigated using the proposed algorithm. A* algorithm is extensively applied in pathfinding and graph traversal.Unlike the other algorithms mentioned above, $A^{*}$ algorithm entails heuristic function to uncover the cost of path that traverses through the particular state. In the structured work $A^{*}$ algorithm is applied to unravel the length of the shortest path by utilizing ranking function from the source node to the destination node. A* algorithm is executed by applying best first search with the help of this search, it greedily decides which vertex to investigate subsequently. $\mathrm{A}^{*}$ is equally complete and optimal if an acceptable heuristic is concerned. The arc lengths in interval valued neutrosophic numbers are defuzzified using the score function.. A numerical example is used to illustrate the proposed approach.
\end{abstract}

Keywords: Heuristic function, Interval Valued Neutrosophic Graph, Score Function,Shortest Path Problem. Destination node, Source node.

\section{Introduction}

In order to overcome the real life situations which could not be handled in some conditions, Zadeh[1] introduced Fuzzy logic which was further developed by Zimmermann[2]. For handling uncertainty the interval valued neutrosophic set is used. The truth-membership, the indeterminacy-membership and the falsity-membership are characterized independently in interval valued neutrosophic set which are able to work with the information's which are conflicting, undetermined, and partial. The rational subdivision of studying the nature, origins, and scope of neutralities, in addition to interface with a variety of ideational spectra is phrased as neutrosophy. The extension of neutrosophic set to neutrosophic offset, underset, and overset was proposed by Smarandache[3]. Bipolar neutrosophic sets, simplified neutrosophic sets, interval valued neutrosophic sets, single valued neutrosophic sets etc are various extension of neutrosophic sets[4,5,6]. The single valued neutrosophic notion is helpful in a range of

DOI: $10.5281 /$ zenodo.4127190

Received: Jun 09, 2020 Accepted: September 03, 2020 
fields, such as the decision making problem, medical diagnosis, etc. Various concepts in graph theory were introduced by combining single valued neutrosophic sets. The single valued neutrosophic graph is the simplification of fuzzy graphs and intuitionistic fuzzy graphs. An interval valued neutrosophic graph oversimplifies the notions of a fuzzy graph, an intuitionistic fuzzy graph, an interval valued fuzzy graph and a single valued neutrosophic graph. The most common topic in research is finding the shortest path of the graph by traversing the edges with different types of algorithms. By using the score function Broumi et. al. [7] proposed an algorithm to solve the neutrosophic shortest path problem where the network arc lengths are represented by interval valued neutrosophic numbers. Multiple labeling is applied for finding shortest path with intuitionstic fuzzy arc length by Jabarulla et.al[8]. Kumar and Kaur [9] provided the solution of fuzzy maximal flow problems using fuzzy linear programming. Garg et al. [10] have proposed the Hybrid model for medical diagnosis using Neutrosophic Cognitive Maps with Genetic Algorithms. An Algorithm for shortest path problem in a network with interval valued intuitionstic trapezoidal fuzzy number was presented by Kumar et al.[11]. Jayagowri et al. [12] used Trapezoidal Intuitionistic Fuzzy Number to Find Optimized Path in a Network. Broumi et al have dealt with various concepts of neutroshopic graphs like single valued neutrosophic graphs, on bipolar single valued neutrosophic graphs and interval valued neutrosophic graphs etc with different algorithms [13,14,15,16,17,18]. Various aspects of Neutrosophic Graphs were studied by Smarandache[19]. Pentagonal Neutrosophic Number and its Application in Networking Problem was proposed by Avishek Chakraborty [20]. Thamaraiselvi et al.[21] found a new approach for optimization of real life transportation problems in neutrosophic environment. Tuhin bera[22] gave an approach to solve the linear programming problem using single valued trapezoidal neutrosophic number. Sapan Kumar Das [23] have used neutrosophic numbers in integer programming. Edalatpanah [24] suggested a new technique to solve triangular neutrosophic linear programming . Majumdar et al.[25] has worked on shortest path problem on intuitionistic fuzzy network . Bhimraj basumatary[26] have unraveled the interval-valued triangular neutrosophic linear programming .There are many algorithms existing for solving the shortest path problems like Dijkstra's, Bellman-Ford,Floyd-Warshall and kruskal's etc for finding the optimal path. In this paper A* algorithm is applied for solving the interval valued neutrosophic shortest path problem.

$\mathrm{A}^{*}$ algorithm is a best-first search algorithm that depends on an open list and a closed list to discover a path that is both optimal and complete towards the goal. $\mathrm{A}^{*}$ search finds the shortest path through a search space to goal state using heuristic function. This technique finds minimal cost solutions and is directed to a goal state called $\mathrm{A}^{*}$ search. This algorithm is complete if the branching factor is finite and every action has fixed cost. By defuzzifying the given interval valued neutrosophic cost by applying score function and by applying $\mathrm{A}^{*}$ algorithm we find the optimal path.

This paper is organized as follows. In Section 2, the basic concepts about neutrosophic sets and interval valued neutrosophic graph is presented. In Section 3, A* algorithm is proposed to find the shortest path and distance in an interval valued neutrosophic graph. In Section 4 a numerical example is illustrated with the algorithm .Section 5 conclusion and proposals for future research is given.

\section{Preliminaries[16]}

Definition 2.1:

Let $\mathrm{X}$ be a space of points with generic elements in $\mathrm{X}$ denoted by $\mathrm{x}$ is the neutrosophic set $\mathrm{A}$ is an object having the form, $\mathrm{A}=\left\{\left\langle x: T_{A}(X), I_{A}(X), F_{A}(X)\right\rangle, x \in X\right\}$, where the functions T,I,F :X $\left.\rightarrow\right]^{-0}, 1^{+}[$define respectively the truthmembership function, indeterminacy- membership function and falsity - membership function of the element, $x \in$ $X$ to the set A with the condition $0 \leq T_{A}(X)+I_{A}(X)+F_{A}(X) \leq 3^{+}$. The functions are real standard or non standard subsets of $]^{-0} 0,1^{+}[$.

\section{Definition 2.2:}

Let $\mathrm{R}_{\mathrm{N}}=\left\langle\left[R_{T}, R_{I}, R_{M}, R_{E},\right]\left(T_{R}, I_{R}, F_{R}\right)\right\rangle$ and $\mathrm{S}_{\mathrm{N}}=\left\langle\left[S_{T}, S_{I}, S_{M}, S_{E},\right]\left(T_{S}, I_{S}, F_{S}\right)\right\rangle$ be two trapezoidal neutrosophic numbers (TpNNs) and $\theta \geq 0$, then

$\mathrm{R}_{\mathrm{N}} \oplus \mathrm{S}_{\mathrm{N}}=\left\langle\left[R_{T}+S_{T}, R_{I}+S_{I}, R_{M}+S_{M}, R_{E}+S_{E}\right]\left(T_{R}++T_{S}-T_{R} T_{S}, I_{R} I_{S}, F_{R} F_{S}\right)\right\rangle$

$\mathrm{R}_{\mathrm{N}} \otimes \mathrm{S}_{\mathrm{N}}=\left\langle\left[R_{T} \cdot S_{T}, R_{I} \cdot S_{I}, R_{M} \cdot S_{M}, R_{E} \cdot S_{E} \quad\right]\left(T_{R} \cdot T_{S}, I_{R}+I_{S}-I_{R} \cdot I_{S}, F_{R}+F_{S}-F_{R} \cdot F_{S}\right)\right\rangle$ 
$\left.\theta \mathrm{R}_{\mathrm{N}}=\left\langle\left[\boldsymbol{\theta} R_{T}, \boldsymbol{\theta} R_{I}, \boldsymbol{\theta} R_{M}, \boldsymbol{\theta} R_{E},\right]\left(1-\left(1-T_{R}\right)\right)^{\theta},\left(I_{R}\right)^{\theta},\left(F_{R}\right)^{\theta}\right)\right\rangle$

Definition 2.3:

Let $\mathrm{X}$ is a space of points (objects) with generic elements in $\mathrm{X}$ denoted by $\mathrm{x}$. An interval valued neutrosophic set $\mathrm{A}$ (INS A) in $\mathrm{X}$ is shown by the truth- membership function $T_{A}(x)$, an indeterminacy-membership function $I_{A}(x)$, and a falsity-membership function $F_{A}(x)$. For each point $\mathrm{x}$ in $\mathrm{X}$, there are $T_{A}(x)=\left[T_{A}^{L}, T_{A}^{U}\right] \subseteq[0,1], I_{A}(x)=$ $\left[I_{A}^{L}, I_{A}^{U}\right] \subseteq[0,1], F_{A}(x)=\left[F_{A}^{L}, F_{A}^{U}\right] \subseteq[0,1]$, and the $\operatorname{sum} T_{A}(x), I_{A}(x)$ and $F_{A}(x)$ satisfies the condition $0 \leq \sup _{A}(x)+\operatorname{supI}_{A}(x)+\sup _{A}(x) \leq 3$, then an INS can be expressed as $\mathrm{A}=\left\{\left\langle x: T_{A}(x), I_{A}(x), F_{A}(x)\right\rangle, x \in\right.$ $X\}=\left\{\left\langle x:\left[T_{A}^{L}, T_{A}^{U}\right],\left[I_{A}^{L}, I_{A}^{U}\right],\left[F_{A}^{L}, F_{A}^{U}\right]\right\rangle, x \in X\right\}$

Definition 2.4:

Let $\widetilde{A}_{1}=\left\langle\left[\boldsymbol{T}_{1}^{L}, \boldsymbol{T}_{1}^{U}\right],\left[\boldsymbol{I}_{1}^{L}, I_{1}^{U}\right],\left[\boldsymbol{F}_{1}^{L}, \boldsymbol{F}_{1}^{U}\right]\right\rangle$ and $\widetilde{A}_{2}=\left\langle\left[\boldsymbol{T}_{2}^{L}, \boldsymbol{T}_{2}^{U}\right],\left[\boldsymbol{I}_{2}^{L}, I_{2}^{U}\right],\left[F_{2}^{L}, \boldsymbol{F}_{2}^{U}\right]\right\rangle$

be two interval valued neutrosophic numbers and $1>0$. Thus, the operational rules are defined as:

$$
\begin{aligned}
& \text { 1. } \tilde{A}_{1} \oplus \tilde{A}_{2}=\left[T_{1}^{L}+T_{2}^{L}-T_{1}^{L} T_{2}^{L}, T_{1}^{U}+T_{2}^{U}-T_{1}^{U} T_{2}^{U}\right] \cdot\left[I_{1}^{L} I_{2}^{L}, I_{1}^{U} I_{2}^{U},\right] \cdot\left[F_{1}^{L} F_{2}^{L}, F_{1}^{U} F_{2}^{U},\right] \\
& \text { 2. } \tilde{A}_{1} \otimes \tilde{A}_{2}=\left\langle\left[T_{1}^{L} T_{2}^{L}, T_{1}^{U} T_{2}^{U},\right] \cdot\left[I_{1}^{L}+I_{2}^{L}-I_{1}^{L} I_{2}^{L}, I_{1}^{U}+I_{2}^{U}-I_{1}^{U} I_{2}^{U}\right] \cdot\left[F_{1}^{L}+F_{2}^{L}-F_{1}^{L} F_{2}^{L}, F_{1}^{U}+F_{2}^{U}-F_{1}^{U} F_{2}^{U}\right]\right\rangle \\
& \text { 3. } \lambda \check{A}=\left\langle\left[1-\left(1-T_{I}^{L}\right)^{\lambda}, 1-\left(1-T_{I}^{U}\right)^{\lambda}\right] \cdot\left[\left(I_{1}^{L}\right)^{\lambda} \cdot\left(I_{1}^{U}\right)^{\lambda}\right] \cdot\left[\left(F_{1}^{L}\right)^{\lambda} \cdot\left(F_{1}^{U}\right)^{\lambda}\right]\right\rangle \\
& \text { 4. } \tilde{A}^{\lambda}=\left\langle\left[\left(T_{1}^{L}\right)^{\lambda},\left(T_{1}^{U}\right)^{\lambda}\right] \cdot\left[1-\left(1-I_{I}^{U}\right)^{\lambda} \cdot 1-\left(1-I_{I}^{U}\right)^{\lambda}\right],\left[1-\left(1-F_{I}^{L}\right)^{\lambda}, 1-\left(1-F_{I}^{U}\right)^{\lambda}\right]\right\rangle \text {, Where } \lambda>0
\end{aligned}
$$

Definition 2.5: To compare between two IVNN, Ridvan [33] used a score function concept in 2014. The score function is used for comparing the IVNS grades. This function demonstrates that the greater the value, the greater the interval-valued neutrosophic sets, and through the use of this concept paths can be ranked.

Let $\tilde{A}_{1}=\left(T_{1}, \mathrm{I}_{1}, \mathrm{~F}_{1}\right)$ be an interval valued neutrosophic number, then, the score function $s\left(\widetilde{\boldsymbol{A}}_{\mathbf{1}}\right)$ of an IVNN can be defined as follows:

$$
\mathrm{S}\left(\hat{A}_{1}\right)=\left(\frac{1}{4}\right) \times\left[2+T_{1}^{L}+T_{1}^{U}-2 I_{1}^{L}-2 I_{1}^{U}-F_{1}^{L}-F_{1}^{U}\right]
$$

Comparison of interval valued neutrosophic numbers

Let $\tilde{A}_{1}=\left(T_{1}, \mathrm{I}_{1}, \mathrm{~F}_{1}\right)$ and $\tilde{A}_{2}=\left(T_{2}, \mathrm{I}_{2}, \mathrm{~F}_{2}\right)$ be two interval valued neutrosophic

numbers then

(i) $\quad \hat{A}_{1}<\hat{A}_{2}$ if $\mathrm{S}\left(\hat{A}_{1}\right)<\mathrm{S}\left(\hat{A}_{2}\right)$

(ii) $\quad \hat{A}_{1}>\hat{A}_{2}$ if $\mathrm{S}\left(\hat{A}_{1}\right)>\mathrm{S}\left(\hat{A}_{2}\right)$

(iii) $\quad \hat{A}_{1}=\hat{A}_{2}$ if $\mathrm{S}\left(\hat{A}_{1}\right)=\mathrm{S}\left(\hat{A}_{2}\right)$

3. Basic notations in $A^{*}$ search

$\mathrm{f}-\mathrm{f}$ is the parameter of $\mathrm{A}^{*}$ which is the sum of the other parameters $\mathrm{G}$ and $\mathrm{H}$ and is the least cost from one node to the next node. This parameter is responsible for helping us find the most optimal path from our source to destination. $\mathrm{g}-\mathrm{g}$ is the cost of moving from one node to the other node. This parameter changes for every node as we move up to find the most optimal path.

$\mathrm{h}-\mathrm{h}$ is the heuristic/estimated path between the current code to the destination node. This cost is not actual but is, in reality, a guess cost that we use to find which could be the most optimal path between our source and destination

\section{1: Algorithm for $A *$ Search}

1. Locate the initial node on the list of ORIGIN.

2. If (ORIGIN is empty) or (ORIGIN $=$ GOAL) terminate search.

3. Remove the first node from ORIGIN. Call this node as $n$.

4. If $(n=$ GOAL $)$ terminate search with success.

5. Else in case, if node a has successors, generate all of them. Find the fitness number of the successors by totaling the evaluation function value $\&$ the cost function value. Sort the list by fitness number.

6. Name the list as START.

7. Replace ORIGIN with START. 


\section{Go to step 2 .}

\section{2: A* Pseudo Code}

Let us have the following assumptions,

Let us denote the goal node as ng, node_current as nc, node_successor as nsc ,successor_current_cost as scc and source node is ns.

The nodes that have been evaluated by the heuristic function but not expanded into successors yet are collected in OPEN set.

The nodes that have been visited and expanded in CLOSE set.

1. Put $\mathrm{ns}$ in the OPEN list with $\mathrm{f}(\mathrm{ns})=\mathrm{h}(\mathrm{ns})$ (initialization)

2. While the OPEN list is not empty \{

3. Take from the open list the node nc with the lowest

4. $\mathrm{f}(\mathrm{nc})=\mathrm{g}(\mathrm{nc}) \oplus \mathrm{h}(\mathrm{nc})$

5. if nc is ng we have found the solution; break

6. Generate each state nsc that come after nc

7. for each nsc of nc \{

8. Set $\mathrm{scc}=\mathrm{g}(\mathrm{nc}) \oplus \mathrm{w}(\mathrm{nc}, \mathrm{nsc})$

9. if ns is in the OPEN list \{

10. if $\mathrm{g}(\mathrm{ns}) \leq \mathrm{scc}$ then go to (line 20$)$

11.\} else if nsc is in the CLOSED list \{

12. if $\mathrm{g}(\mathrm{nsc}) \leq \mathrm{scc}$ then go to (line 20$)$

13. Move nsc from the CLOSED list to the OPEN list

14.\} else \{

15. Add nsc to the OPEN list

16. Set $\mathrm{h}(\mathrm{nsc})$ to be the heuristic distance to $\mathrm{ng}$

17.\}

18. Set $\mathrm{g}(\mathrm{nsc})=\mathrm{scc}$

19. Set the parent of nsc to nc

20$.

21. Add nc to the CLOSED list.

22.\}

23. if(nc != ng) exit with error (the OPEN list is empty)

\section{Numerical Example}

Consider the given interval valued neutroshopic shortest path problem with five edges with interval valued neutroshopic fuzzy weights as in Fig:1.

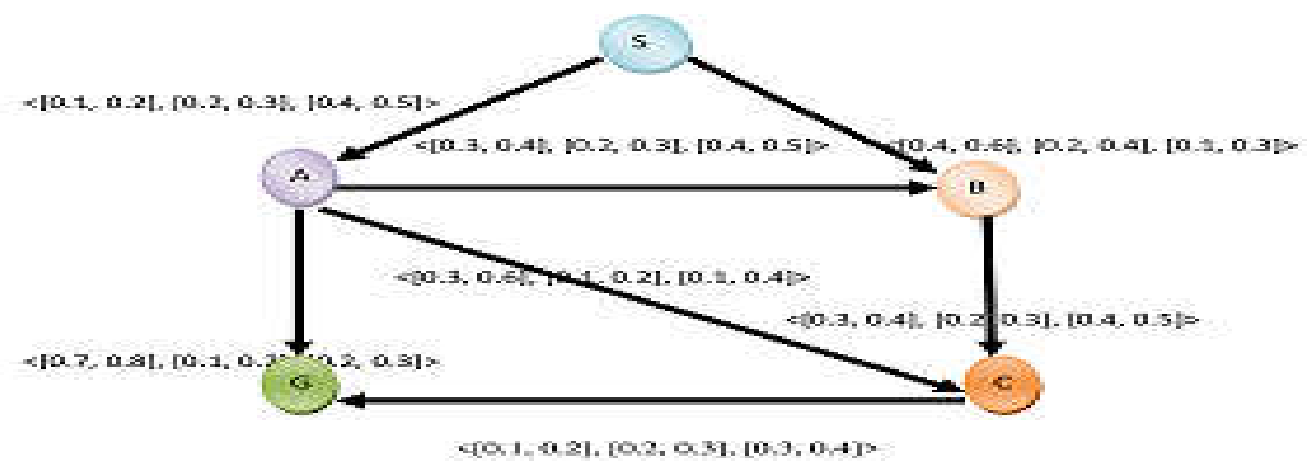


Fig.1.An interval valued neutroshopic shortest path problem

Let us take the interval valued neutroshopic fuzzy weight for the edge from S-A, by applying the score function formula we convert the interval valued neutroshopic fuzzy weights to crisp number,

$<[0.1,0.2],[0.2,0.3],[0.4,0.5]>$

$\mathrm{S}\left((A)=\left(\frac{1}{4}\right) \times\left[2+T_{1}^{L}+T_{1}^{U}-2 I_{1}^{L}-2 I_{1}^{U}-F_{1}^{L}-F_{1}^{U}\right]\right.$

$=\left(\frac{1}{4}\right) \times[2+0.1+0.2-2 \times 0.2-2 \times 0.3-0.4-0.5]$

$=\left(\frac{1}{4}\right) \times[2.3-0.4-0.6-0.4-0.5]$

$=\left(\frac{1}{4}\right) \times[0.4]=0.1$

Similarly by proceeding with the formula for score function, we can find the crisp values given in the table below.

Table:1. Interval valued Neutrosophic distance

\begin{tabular}{|l|c|c|}
\hline Edges & Interval valued Neutrosophic distance & $\begin{array}{l}\text { Crisp } \\
\text { Values }\end{array}$ \\
\hline S-A & $<[0.1,0.2],[0.2,0.3],[0.4,0.5]>$ & 0.1 \\
\hline S-B & $<[0.4,0.6],[0.2,0.4],[0.1,0.3]>$ & 0.35 \\
\hline A-B & $<[0.3,0.4],[0.2,0.3],[0.4,0.5>$ & 0.2 \\
\hline B-C & $<[0.3,0.4],[0.2,0.3],[0.4,0.5>$ & 0.2 \\
\hline A-C & $<[0.3,0.6],[0.1,0.2],[0.1,0.4]>$ & 0.45 \\
\hline A-G & $<[0.7,0.8],[0.1,0.2],[0.2,0.3]>$ & 0.6 \\
\hline C-G & $<[0.1,0.2],[0.2,0.3],[0.3,0.4]>$ & 0.15 \\
\hline
\end{tabular}

After finding the crisp values and substituting in the corresponding paths we get,

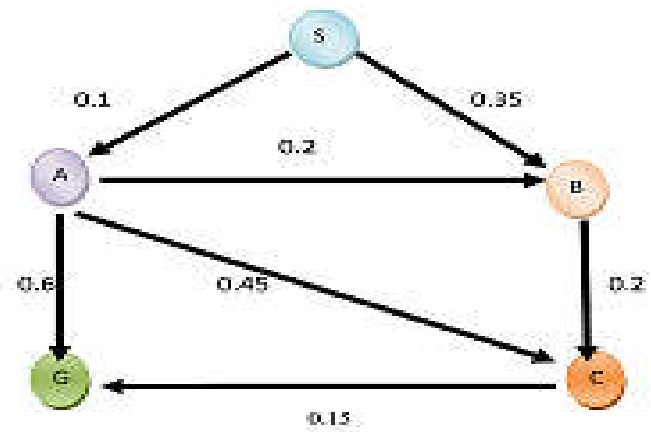

Fig.2. Crisp valued neutroshopic shortest path problem

Interval valued neutroshopic heuristic values to end nodes are given in the following table.2. 
Table: 2 . Heuristic values

\begin{tabular}{|l|c|c|}
\hline Node & h(n) & Crisp h(n) \\
\hline $\mathbf{S}$ & $<[0.9,0.8],[0.1,0.2],[0.2,0.3]>$ & 0.65 \\
\hline $\mathbf{A}$ & $<[0.7,0.8],[0.1,0.2],[0.2,0.3]>$ & 0.6 \\
\hline $\mathbf{B}$ & $<[0.3,0.4],[0.2,0.3],[0.4,0.5>$ & 0.2 \\
\hline $\mathbf{C}$ & $<[0.1,0.2],[0.2,0.3],[0.4,0.5]>$ & 0.1 \\
\hline $\mathbf{G}$ & $<[0,0],[0,0],[1,1]>$ & 0 \\
\hline
\end{tabular}

Let us start with the source node $\mathrm{S}$

\section{ITERATION: 0}

\section{$\mathrm{S} \rightarrow 0 \oplus 0.65=0.65$}

From the source node $\mathrm{S}$ the graph expands through two paths A and B.

\section{ITERATION: 1}

$\mathrm{S} \rightarrow \mathrm{A}: \mathrm{f}(\mathrm{A})=\mathrm{g}(\mathrm{A}) \oplus \mathrm{h}(\mathrm{A})=0.1 \oplus 0.6=0.7$

$\mathrm{S} \rightarrow \mathrm{B}: \mathrm{f}(\mathrm{A})=\mathrm{g}(\mathrm{B}) \oplus \mathrm{h}(\mathrm{B})=0.35 \oplus 0.2=0.55(\mathrm{MIN})$

By comparing the above values the path $\mathrm{S} \rightarrow \mathrm{B}$ has minimum value, so we proceed to traverse from that path,

ITERATION: 3

$\mathrm{S} \rightarrow \mathrm{B} \rightarrow \mathrm{C}: \mathrm{f}(\mathrm{A})=\mathrm{g}(\mathrm{C}) \oplus \mathrm{h}(\mathrm{C})=(0.35 \oplus 0.2) \oplus 0.1=0.65$

$\mathrm{S} \rightarrow \mathrm{A} \rightarrow \mathrm{B}: \mathrm{f}(\mathrm{B})=\mathrm{g}(\mathrm{B}) \oplus \mathrm{h}(\mathrm{B})=(0.1 \oplus 0.2) \oplus 0.2=0.5(\mathrm{MIN})$

$\mathrm{S} \rightarrow \mathrm{A} \rightarrow \mathrm{C}: \mathrm{f}(\mathrm{C})=\mathrm{g}(\mathrm{C}) \oplus \mathrm{h}(\mathrm{C})=(0.1 \oplus 0.45) \oplus 0.1=0.65$

$\mathrm{S} \rightarrow \mathrm{A} \rightarrow \mathrm{G}: \mathrm{f}(\mathrm{G})=\mathrm{g}(\mathrm{G}) \oplus \mathrm{h}(\mathrm{G})=(0.1 \oplus 0.6) \oplus 0=0.7$

By comparing the above values the path $\mathrm{S} \rightarrow \mathrm{A} \rightarrow \mathrm{B}$ has minimum value, so we proceed to traverse from that path,

\section{ITERATION: 4}

$\mathrm{S} \rightarrow \mathrm{A} \rightarrow \mathrm{B} \rightarrow \mathrm{C}: \mathrm{f}(\mathrm{C})=\mathrm{g}(\mathrm{C}) \oplus \mathrm{h}(\mathrm{C})=(0.1 \oplus 0.2 \oplus 0.2) \oplus 0.1=0.6(\mathrm{MIN})$

By comparing the paths $\mathrm{S} \rightarrow \mathrm{A} \rightarrow \mathrm{B} \rightarrow \mathrm{C}$ and $\mathrm{S} \rightarrow \mathrm{B}$ the path $\mathrm{S} \rightarrow \mathrm{B}$ is minimum.

So traverse from that path to reach the goal node.

\section{ITERATION: 5}

$\mathrm{S} \rightarrow \mathrm{B} \rightarrow \mathrm{C} \rightarrow \mathrm{G}: \mathrm{f}(\mathrm{G})=\mathrm{g}(\mathrm{G}) \oplus \mathrm{h}(\mathrm{G})=(0.35 \oplus 0.2 \oplus 0.15) \oplus 0=0.7$

By comparing the paths $\mathrm{S} \rightarrow \mathrm{B} \rightarrow \mathrm{C} \rightarrow \mathrm{G}$ and $\mathrm{S} \rightarrow \mathrm{A} \rightarrow \mathrm{B} \rightarrow \mathrm{C}$ the

path $\mathrm{S} \rightarrow \mathrm{A} \rightarrow \mathrm{B} \rightarrow \mathrm{C}$ is minimum.

So traverse from that path to reach the goal node.

\section{ITERATION: 6}

To reach the goal node we can traverse in the two paths,

$\mathrm{S} \rightarrow \mathrm{A} \rightarrow \mathrm{B} \rightarrow \mathrm{C} \rightarrow \mathrm{G}: \mathrm{f}(\mathrm{G})=\mathrm{g}(\mathrm{G}) \oplus \mathrm{h}(\mathrm{G})=(0.1 \oplus 0.2 \oplus 0.2 \oplus 0.15) \oplus 0=0.65(\mathrm{MIN})$

$\mathrm{S} \rightarrow \mathrm{A} \rightarrow \mathrm{C} \rightarrow \mathrm{G}: \mathrm{f}(\mathrm{G})=\mathrm{g}(\mathrm{G}) \oplus \mathrm{h}(\mathrm{G})=(0.1 \oplus 0.45 \oplus 0.15) \oplus 0=0.7$

By comparing the paths $\mathrm{S} \rightarrow \mathrm{A} \rightarrow \mathrm{B} \rightarrow \mathrm{C} \rightarrow \mathrm{G}$ and $\mathrm{S} \rightarrow \mathrm{A} \rightarrow \mathrm{C} \rightarrow \mathrm{G}$ the path $\mathrm{S} \rightarrow \mathrm{B} \rightarrow \mathrm{C} \rightarrow \mathrm{G}$ is minimum 


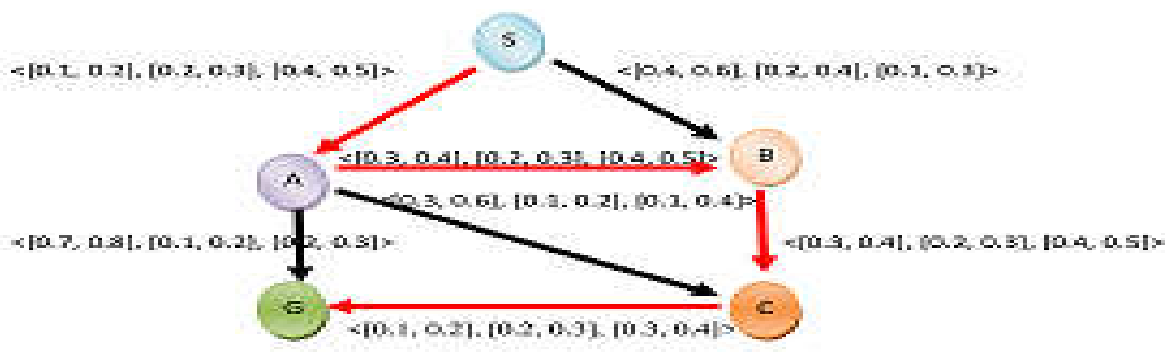

Fig.3. Shortest path from source node to goal node.

The shortest path is given by $\mathbf{S} \rightarrow \mathbf{A} \rightarrow \mathbf{B} \rightarrow \mathbf{C} \rightarrow \mathbf{G}$.

\section{Conclusion}

$\mathrm{A}^{*}$ algorithm is applied to solve the shortest path problem on a network with an interval valued neutrosophic arc lengths in this paper. $A^{*}$ algorithm is complete and optimal. $A^{*}$ algorithm is the best one from other techniques. It is used to solve very complex problems. A* algorithm is optimally efficient, i.e. there is no other optimal algorithm guaranteed to expand fewer nodes than $\mathrm{A}^{*}$.Heuristic values are considered in calculating the path by this method. Score function is used for defuzzification. The technique is enlightened by a numerical example with the help of theoretical information. The time complexity of $A^{*}$ depends on the heuristic. In the worst case of an unbounded search space, the number of nodes expanded is exponential in the depth of the solution (the shortest path) $d: O\left(b^{d}\right)$, where $b$ is the branching factor. $A^{*}$ can also be adapted to a bidirectional search algorithm.Furthermore, the following algorithm of the interval neutrosophic shortest path problem can be extended into an interval valued bipolar neutrosophic environment.

Funding: "This research received no external funding"

Conflicts of Interest: "The authors declare no conflict of interest."

\section{References}

1. L.Zadeh, "Fuzzy sets", Inform and Control, 8, 338-353, 1965.

2. H. J. Zimmermann, "Fuzzy set Theory - and Its Applications", Kluwer-Nijhoff Publishing, BostonDordrecht-Lancaster, 1985.

3. Florentin Smarandache, "Neutrosophic Overset, Neutrosophic Underset, and Neutrosophic Offset". Similarly for Neutrosophic Over-/Under-/Off- Logic, Probability, and Statistics, 168 p., Pons Editions, Bruxelles, Belgique.2016.

4. S.Broumi, A.Bakali, M.Talea, F.Smarandache, “On Bipolar Single Valued Neutrosophic Graphs”, Journal of New Theory, N11, pp.84-102, 2016.

5. S.Broumi, A,Bakali, M.Talea, F.Smarandache " Single Valued Neutrosophic Graphs", Journal of New 
Theory, N 10, pp. 86-101,2016.

6. S.Broumi, A. Bakali, M.Talea, F.Smarandache, "Single Valued Neutrosophic Graphs: Degree, Order and Size”, IEEE International Conference on Fuzzy Systems (FUZZ), pp.2444-2451,2016.

7. S.Broumi, A,Bakali, M.Talea, F.Smarandache, L.Vladareanu, "Computation of Shortest Path Problem in a Network with SV-Trapezoidal Neutrosophic Numbers", Proceedings of the 2016 International Conference on Advanced Mechatronic Systems, Melbourne, Australia, pp.417-422,2016.

8. A.Ngoor and M.Jabarulla , "Multiple labeling Approach For Finding shortest Path with Intuitionstic Fuzzy Arc Length", International Journal of Scientific and Engineering Research,V3,Issue 11,pp.102$106,2012$.

9. A.Kumar and M.Kaur, "Solution of fuzzy maximal flow problems using fuzzy linear programming”, World Academy of Science and Technology.87,28-31,2011.

10. Gaurav, \& Kumar, Megha \& Bhutani, Kanika \& Aggarwal, Swati, "Hybrid model for medical diagnosis using Neutrosophic Cognitive Maps with Genetic Algorithms”. 1-7. 10.1109/FUZZ-IEEE.2015.7338015,2015.

11. G.Kumar, R.K.Bajaj and N.Gandotra, "Algoritm for shortest path problem in a network with interval valued intuitionstic trapezoidal fuzzy number”, Procedia Computer Science, 70, pp.123-129, 2015.

12. P.Jayagowri and G.Geetha Ramani, "Using Trapezoidal Intuitionistic Fuzzy Number to Find Optimized Path in a Network", Advances in Fuzzy Systems., Article ID 183607, 6 pages http://dx.doi.org/10.1155/2014/183607,2014.

13. S.Broumi, A,Bakali, M.Talea, F.Smarandache and L.Vladareanu, "Applying Dijkstra for Solving Neutrosophic Shortest Path Problem”, Proceedings of the 2016 International Conference on Advanced Mechatronic Systems, Melbourne, Australia, pp.412-416,2016.

14. S.Broumi, F.Smarandache, "New distance and similarity measures of interval neutrosophic sets, Information Fusion (FUSION)", IEEE 17th International Conference, pp 1-7,2014.

15. S.Broumi, M.Talea, A.Bakali, F.Smarandache, "An Introduction to Bipolar Single Valued Neutrosophic Graph Theory”. Applied Mechanics and Materials, vol.841, 184-191.2016.

16. S.Broumi, M.Talea, A.Bakali, F.Smarandache, K. P.Kishore,Şahin, “ Shortest Path Problem under Interval Valued Neutrosophic Setting”, International Journal of Advanced Trends in Computer Science and Engineering, volume 8,pg216-222,2019.

17. S.Broumi, A.Bakali, M.Talea, F.Smarandache, "Isolated Single Valued Neutrosophic Graphs". Neutrosophic Sets and Systems, Vol. 11, pp.74-78, 2016.

18. S.Broumi, A.Bakali, M.Talea, F.Smarandache, "Interval Valued Neutrosophic Graphs", Critical Review, XII, pp.5-33, 2016.

19. F.Smarandache, "Types of Neutrosophic Graphs and neutrosophic AlgebraicStructures together with their Applications in Technology", seminar, Universitatea Transilvania din Brasov, Facultatea de Design de Produs si Mediu, Brasov, Romania .2015.

20. Avishek Chakraborty, "A New Score Function of Pentagonal Neutrosophic Number and its Application in Networking Problem”, International Journal of Neutrosophic Science, Volume 1, Issue 1, PP: 40-51, 2020. 
21. R.Thamaraiselvi and R. Santhi, "A New Approach for Optimization of Real Life Transportation Problems in Neutrosophic Environment", Mathematical Problems in Enginering.Article ID 5950747,9http://dx.doi.org/10.1155/2016/5950747,2016.

22. Tuhin Bera, Nirmal Kumar Mahapatra, “An Approach to Solve the Linear Programming Problem Using Single Valued Trapezoidal Neutrosophic Number", International Journal of Neutrosophic Science ,Vol. 3, No. 2, PP. 54-66, 2020.

23. Sapan Kumar Das, S.A. Edalatpanah, “A new ranking function of triangular neutrosophic number and its application in integer programming", International Journal of Neutrosophic Science, Volume 4 , Issue 2, PP: $82-92,2020$.

24. S.A.Edalatpanah, "A Direct Model for Triangular Neutrosophic Linear Programming”, International Journal of Neutrosophic Science Volume 1, Issue 1, PP: 19-28, 2020.

25. S.Majumdar and A.Pal, "Shortest Path Problem on Intuitionistic Fuzzy Network", Annals of Pure and Applied Mathematics, Vol. 5, 1, 26-36, 2013.

26. Bhimraj Basumatary, Said Broumi, “Interval-Valued Triangular Neutrosophic Linear Programming Problem", International Journal of Neutrosophic Science, Volume 10, Issue 2, PP: 105-115, 2020. 